Stéphane Méresse

Ulrike Bauer

Thomas Ludwig Fabienne Mauxion

Anne Schmidt Bernard Hoflack

\section{Bases moléculaires du transport vers les lysosomes}

Chez les eucaryotes supérieurs, les récepteurs du mannose 6-phosphate sont des composants essentiels qui permettent le ciblage des hydrolases acides vers les lysosomes. Deux récepteurs ont été caractérisés. Le premier lie les enzymes lysosomiales (dont les oligosaccharides portent des résidus de mannose 6-phosphate) et l'insulin-like growth factor II, un facteur de croissance non glycosylé. Le second a une spécificité de liaison restreinte aux hydrolases. Les récepteurs sont recyclés en permanence entre différents compartiments cellulaires. Dans le réseau trans-golgien, ils assurent le tri des enzymes lysosomiales qu'ils déversent ensuite dans les endosomes. Ils recyclent également entre la membrane plasmique et les endosomes pour internaliser les ligands extracellulaires. Pour assurer ces fonctions, ils utilisent des séquences signal de leurs domaines cytoplasmiques, qui sont reconnues par des protéines cytosoliques et permettent leur empaquettage sélectif dans des vésicules de transport.

\section{ADRESSE}

S. Méresse : chercheur post-doctoral, boursier $C E E$. U. Bauer : technicienne EMBL. T. Ludwig : chercheur post-doctoral, boursier Bæhringer Ingelheim. F. Mauxion : chercheur post-doctoral, boursière ATIPE/ARC. A. Schmidt : chercheur post-doctoral, boursière EMBO. B. Hoflack : directeur de recherche au Cnrs, chef de groupe à l'EMBL. Laboratoire européen de biologie moléculaire (EMBL), département de biologie cellulaire, Meyerhof strasse, 1, Postfach es lysosomes sont l'un des principaux sites de la dégradation cellulaire. Ils ont pour fonction de cataboliser et de recycler un éventail rès large de molécules d'origine endogène (autophagie des composants cellulaires) ou exogène (par exemple, phagocytose de bactéries et endocytose d'hormones ou de lipoprotéines). Les lysosomes possèdent pour cela un éventail de plus de 40 hydrolases acides (protéases, glycosidases, lipases, nucléases, etc.) qui fonctionnent de concert pour dégrader les molécules en éléments simples, directement réutilisables. Le bon fonctionnement des lysosomes est un élément essentiel du métabolisme. Un dysfonctionnement lysosomial entraîne l'accumulation de macromolécules ou d'intermédiaires métaboliques. Les manifestations cliniques de ces dysfonctionnements sont variées et souvent sévères. Ces affections sont regroupées sous le terme générique de maladies lysosomiales.

\section{Les maladies Iysosomiales}

Ces maladies héréditaires sont en général provoquées par l'absence d'une activité enzymatique due à un défaut de synthèse ou à l'instabilité de la protéine. Dans certains cas, un défaut de transport vers les lysosomes provoque l'absence de la presque totalité des hydrolases [1-3]. On 
recense aujourd'hui plus de trente maladics lysosomiales, classées en fonction de la nature du substrat non dégradé. Citons, par exemple, les mucopolysaccharidoses (accumulation de glycosaminoglycanes), les lipidoses ou les mucolipidoses. Un déficit enzymatique affecte l'ensemble de l'organisme, mais les cellules les plus touchées sont celles dans lesquelles le substrat de l'enzyme est abondant. La maladie de Tay-Sachs se caractérise ainsi par l'absence de dégradation des gangliosides, dont le renouvellement est particulièrement rapide dans le cerveau. En conséquence, les patients présentent des troubles du système nerveux central [4].

$\mathrm{Si}$ les recherches effectuées sur ces maladies ont permis de comprendre leurs bases génétiques, elles ont aussi dévoilé les mécanismes moléculaires impliqués dans le ciblage des hydrolases vers les lysosomes. C'est dans les années 1970 que le groupe de Neufeld [2], étudiant les maladies de Hunter/Hurler et les mucolipidoses II, montra que ce ciblage met en jeu un signal commun à toutes les hydrolases ct des récepteurs spécifiques. Ce signal fut identifié aux résidus de mannose 6-phosphate (M6P), pré- sents sur la partie oligosaccharidique de cette classe de protéines. Les récepteurs du mannose 6-phosphate, qui assurent le transport des enzymes lysosomiales vers les lysosomes, furent ensuite caractérisés.

\section{Biosynthèse du signal mannose 6-phosphate}

Les enzymes lysosomiales et les glycoprotéines de sécrétion sont synthétisées par les polysomes liés aux membranes du réticulum endoplasmique (RE). Au cours de leur translocation dans la lumière de cet organite, ces polypeptides sont glycosylés sur certains résidus d'asparagine par addition d'oligosaccharides de type oligomannosidique préassemblés sur un lipide (figure 1). Après un premier épissage de l'oligosaccharide (hydrolyse des résidus de glucose et d'un mannose), ces protéines sont vectoriellement transportées vers l'appareil de Golgi où leurs oligosaccharides sont modifićs. La modification spécifique des hydrolases nécessite l'action concertée de deux enzymes. La première, la $\mathrm{N}$-acétylglucosamine-1-phosphotransférase ou phosphotransférase, probablement localisée dans le réticu- lum cndoplasmique et le cis-Golgi, élabore un intermédiaire phosphodicster entre le carbone 6 du mannose et un résidu $\mathrm{N}$-acétylglucosamine 1-phosphate (pour revue, voir [5]). Cette enzyme a une très hautc affinité pour les hydrolases $(\mathrm{Km} \simeq$ $10,20 \mu \mathrm{M})$. La seconde, la phosphodiester $\alpha$-N-acétylglucosaminidase, probablement localisée dans le medium-Golgi, hydrolyse les résidus $\mathrm{N}$-acétylglucosamine et laisse ainsi apparaître des résidus phosphomonoester (M6P) qui permettront la liaison ultérieure des hydrolases aux récepteurs. Les autres glycoprotéines, dont les oligosaccharides ne sont pas phosphorylés mais convertis en structures complexes (figure 1), ne peuvent se lier aux récepteurs et sont sécrétées par défaut dans le milieu extracellulaire. Dans les mucolipidoses II ct III, la phosphotransférase n'est pas fonctionnelle et les enzymes lysosomiales, non phosphorylées, sont donc sécrétées.

Ces enzymes clés du ciblage, en particulier la phosphotransférase, sont encore mal connues: elles ne sont pas purifiées, et leurs ADNc ne sont pas clonés. Certaines études réalisées sur des fibroblastes de patients

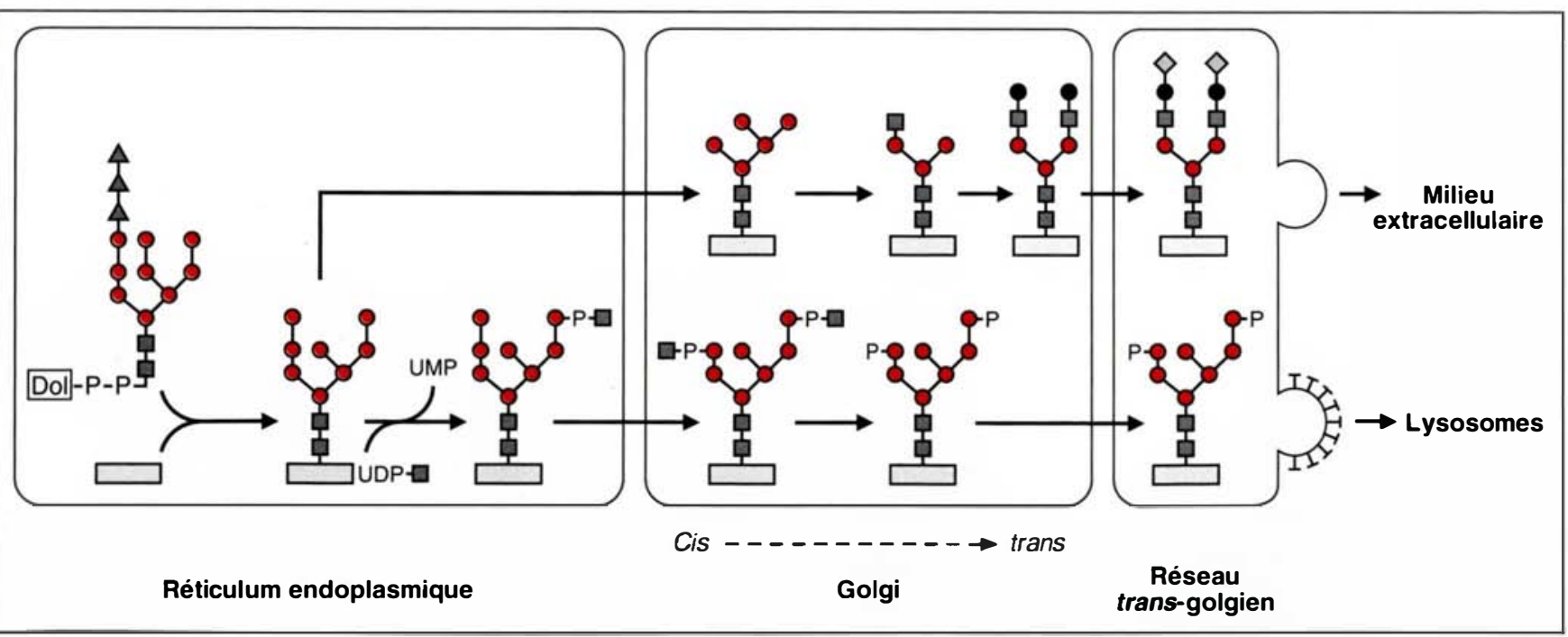

Figure 1. Glycosylation des enzymes lysosomiales et des protéines de sécrétion. La glycosylation de résidus asparagine débute dans le réticulum endoplasmique par l'addition d'un oligosaccharide préassemblé sur le dolichol pyrophosphate (DolPP). Après épissage de l'oligosaccharide, la phosphotranférase catalyse le transfert de $\mathrm{N}$-acétylglucosamine 1-phosphate à partir de I'UDP-N-acétylglucosamine sur certains résidus de mannose des oligosaccharides des hydrolases. Une $\mathrm{N}$-acétylgucosaminidase spécifique laisse apparaître les signaux mannose 6-phosphate. Les oligosaccharides des autres protéines de sécrétion sont transformés en structures de type $\mathrm{N}$-acétyllactosaminique. Cette représentation rend compte de la localisation probable des différentes enzymes impliquées dans ces modifications. acétylglucosamine; $\mathbf{0}$, mannose; $\mathbf{\Delta}$, glucose ; 0 , galactose ; 0 , acide sialique.

$m / s n^{\circ} 2$ vol. 9, févier 93 


\section{RÉFÉRENCES}

1. Ncufled EF, Lim TW, Shapiro LJ. Inherited disorders of lysosomal metabolism. Ann Rev Biochem 1975; 44: 357-76.

2. Neufcld EF. Lysosomal storage diseases. Ann Rev Biochem 1991; 60 : 257-80.

3. Crine $\mathrm{P}$, Des Parois L, Lecavalier $\mathrm{H}$ Un code postal pour les enzymes lysosomiales. médecine/sciences $1987 ; 3: 453-60$.

4. Dreyfus JC, Akli S, Pocnaru L. Maladies de Tay-Sachs et de Sandhoff : les déficits en $\beta$-hexosaminidases, modèles de maladies des lysosomes. médecine/sciences 1992; 8 797-804.

5. Kornfeld S, Mellman 1. The biogenesis of lysosomes. Ann Rev Cell Biol 1989; 5 : 483-525.

6. Baranski 'TJ, Koclsch G, Hartsuck JA, Kornfeld S. Mapping and molecular modelling of a recognition domain for lysosomal cnzyme targeting. J Biol Chem 1991; 266 . 23365-72.

7. Dahms NM, Lobcl P, Kornfeld S. Mannose 6-phosphate receptors and lysosomal enzymc targeting. J Biol Chem 1989; 264 : 12115-8.

8. Hofack B, Lobel P. Functions of the mannose 6-phosphatc reccptors. In : Storrie B, Murphy R, eds. Endosomes and Lysosomes: a Dynamic Relationship. Grcenwich, CT : JAI Press, 1993 (sous presse).

9. Sahagian GG, Distler J, Jourdian JW. Characterization of a membrane-associated receptor from bovine liver that binds phosphomannosyl residues of bovine testicular $\beta$ galactosidasc. Proc Natl Acad Sci USA 1981; $78: 4289-93$.

10. Hoflack B, Kornfeld S. Purification and characterization of a cation-dependent mannose 6-phosphatc receptor from murine P388D1 macrophages and bovine liver. $J$ Biol Chem 1985 ; 260 : 12008-14.

11. Roth RA. The structure of the receptor for insulinc-like growth factor II : the puzzlc amplified. Science 1988; 239 : 1269-71. atteints de mucolipidose III, suggèrent que la phosphotransférase est formée d'au moins deux sous-unités : l'une, spécialisée dans la reconnaissance de la partic protéique du substrat ; l'autre, catalytique, assure la phosphorylation de la partie saccharidique. Un aspect fascinant de ces études concerne la compréhension des bases moléculaires et structurales permettant à la phosphotransférase de reconnaître les hydrolases. Il est clair que ces interactions sont de type protéinc-protéine puisque les oligosaccharides sont de très mauvais substrats $(\mathrm{Km} \simeq 20 \mathrm{mM})$. Les $\mathrm{ADNc} d \mathrm{de}$ plus de dix enzymes lysosomiales ont été clonés, mais la comparaison des séquences primaires n'a révélé aucun motif commun susceptible d'interagir avec la phosphotransférase. Ce motif est donc à rechercher dans la structure tridimensionnelle de cette classe de protéines. Des études récentes [6], utilisant comme modèle des chimères de la cathcpsine D (dirigée vers les lysosomes) et du pepsinogène (protéine sécrétée et structurellement homologue à la cathepsine D), ont montré que certains acides aminés, en particulier des résidus de lysine, dispersés dans la séquence primaire de la protéine mais se regroupant dans sa structure tridimensionnelle, forment un domaine potentiel de reconnaissance (figure 2). Cela reste néanmoins à confirmer à l'aide d'autres modèles expérimentaux.

\section{Les récepteurs du man- nose 6-phosphate}

Dcux récepteurs capables de lier le signal M6P ont été caractérisés (pour revue, voir $[5,7,8])$. Tous deux sont des glycoprotéines transmembranaires de type $\mathrm{I}^{*}$ de 275 [9] et $46 \mathrm{kDa}$ [10]. Lc second est probablement un homodimère (figure 3 ) et, contrairement au premier, lie plus efficacement son ligand en présence de cations bivalents [10]. Ces protéines

\footnotetext{
* Glycoprotéine ayant un domaine N-terminal extracytoplasmique et un domaine C-terminal cytoplasmique.
}

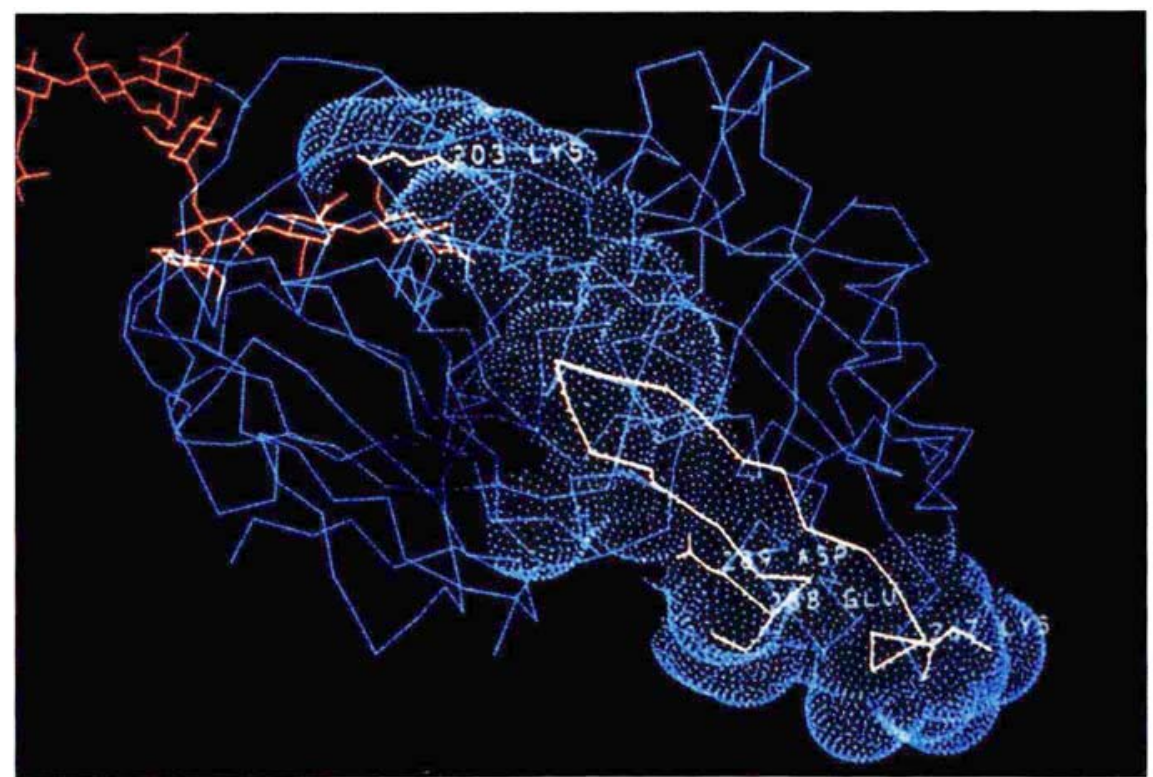

Figure 2. Reconnaissance de la cathepsine D par la phosphotransférase. L'expression de chimères de la cathepsine $D$ et du pepsinogène a permis de prédire la structure du domaine potentiel d'interaction avec la phosphotransférase. Cette figure représente un modèle informatique de cette chimère et montre les acides aminés nécessaires à la phosphorylation des résidus de mannose. Ceux-ci forment une large surface convexe qui s'étend de la lysine 203 jusqu'aux résidus chargés (Lys 267, Glu 288, Asp ${ }^{289}$ ) qui forment la base d'une boucle $\beta$ ( $\beta$ loop, en jaune). Les oligosaccharides apparaissent en orange. (D'après [6].) 
sont donc couramment appelées " récepteur M6P indépendant des cations" ou "grand récepteur" et " récepteur dépendant des cations" ou "petit récepteur". Tous deux ont une très forte affinité pour les oligosaccharides de type oligomannosidique comportant deux groupements phosphomonoester $(\mathrm{Kd} \simeq 2$ et 200 $\mathrm{nM}$, respectivement). Le grand récepteur possède deux sites de liaison pour le mannose 6-phosphate, le petit en possède un par sous-unité. Chaque récepteur pcut donc fixer une hydrolase. Aucune fixation préférentielle d'hydrolases sur l'un ou l'autre des récepteurs n'a pu être observée. Les récepteurs fixent leur ligand à un $\mathrm{pH}$ analogue à celui du réseau transgolgien $(\mathrm{pH} \simeq 6-6,5)$ et le relargue au $\mathrm{pH}$ des endosomes $(\mathrm{pH} \simeq 5)$.

Les séquences comparées des deux récepteurs (pour revue, voir $[5,7,8]$ ) ont révélé certaines homologies de structurc dans leur domaine extracytoplasmique responsable de la fixation du ligand. Celui du grand récepteur (2 269 acides aminés) cst organisé en 15 unités répétitives contiguës d'environ 147 résidus (figure 3). Les unités fixant le M6P n'ont pas cncore été déterminées. Le domaine extracytoplasmique du petit réccptcur (159 acides aminés) est très similaire à chacune des unités répétitives du grand récepteur. Ccla suggère que les deux réceptcurs proviennent d'un même gène ancestral. Les domaines transmembranaires et cytoplasmiques ne montrent pas d'homologies de séquence évidentes. Cela peut paraître surprenant pour des protéines suivant des voies de transport apparemment identiques. Il est cependant probable que des homologies existent dans la structure tridimensionnelle de leur domaine cytoplasmique.

\section{Le grand récepteur du M6P est aussi celui de I'IGF-II}

Le grand récepteur M6P est, tout au moins chez les mammiferes, une molécule bifonctionnelle (pour revue, voir [11]). En effet, le clonage de l'ADNc codant pour le récepteur de l'insulin-like growth factor-II (IGF-II) humain révéla une protéine identique au grand récepteur du M6P [12]. L'IGF-II, est un polypeptide non $m / s n^{\circ} 2$ vol. 9, février 93 glycosylé dont le rôle dans le développement n'est pas encore bien compris. Il se lie sur un site distinct ( $\mathrm{Kd}$ $\simeq 0,2 \mathrm{nM}$ ), mais néanmoins proche de ceux destinés aux résidus M6P (pour revue, voir [5, 8, 13]).

La signification physiologique de cette double fonction reste cependant difficile à établir. En effet, la stimulation de ccllules par l'IGF II ne modi- fie pas le transport intracellulaire des ligands phosphorylés. Le rôle du grand réccpteur dans la dégradation de l'IGF II et la transmission d'un signal a donc été évoqué (pour revue, voir [13]). Ce récepteur ne possède pas d'activité kinase intrinsèque (comme, par exemple, ceux de l'EGF ou de l'insuline) mais pourrait s'associer à des protéines $G$ trimériques

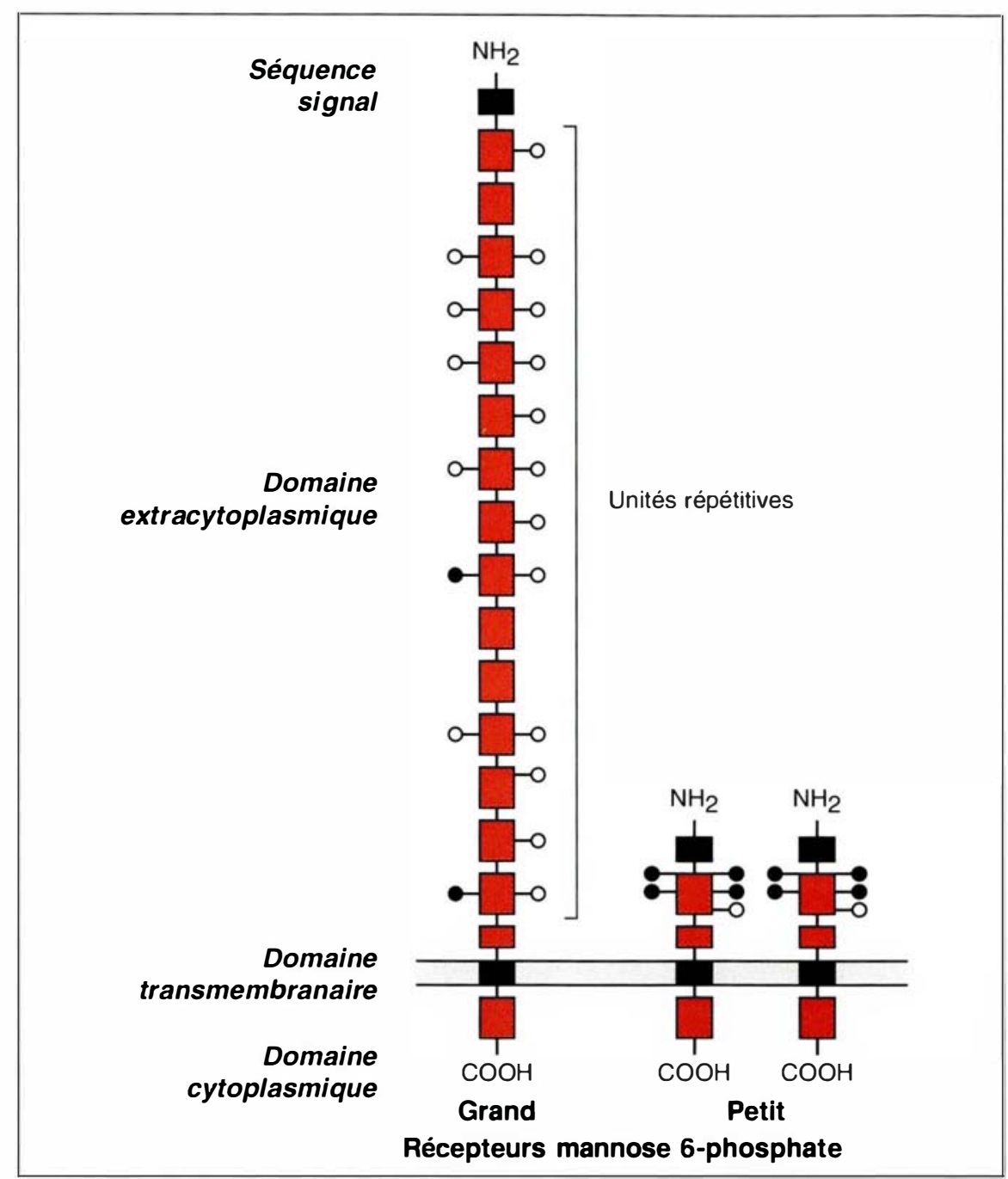

Figure 3. Les récepteurs du mannose 6-phosphate. Le grand récepteur et le petit récepteur bovin (respectivement de 300 et $46 \mathrm{kDa}$ ) sont des glycoprotéines de type 1 formées d'une séquence signal amino-terminale 144 et 28 acides aminés), d'un domaine extracytoplasmique fixant le ligand $(2269$ et 159 résidus), d'un domaine transmembranaire (23 et 25 résidus) et d'un domaine cytoplasmique carboxyterminal (163 et 67 acides aminés). Le domaine extracytoplasmique du grand récepteur est constitué de 15 unités répétitives présentant entre elles des homologies de séquence $(16$ à $38 \%)$. Le domaine extracytoplasmique du petit récepteur est homologue à chacune des unités répétitives du grand récepteur. L'unité fonctionnelle du petit récepteur du M6P est probablement un homodimère. $O$, sites de $\mathrm{N}$-glycosylation connus; $O$, sites de $\mathrm{N}$-glycosylation possibles. (D'après [7].) 


\section{RÉFÉRENCES}

12. Morgan DO, Edman JC, Strandring $\mathrm{DN}$, et al. Insulin-like growth factor II receptor as a multifunctional binding protcin. Nature $1987 ; 32: 301-7$

13. Von Figura K. Molecular recognition and targeting of lysosomal proteins. Curr $O P$ Cell Biol 1991; 3 : 642-6.

14. Okamoto T, Katada T, Murayama Y, Ui M, Ogata E, Nishimoto I. A simple structure encodes G-protcin-activating function of the IGF II/mannose 6-phosphate reccptor. Cell 1990; 62 : 709-17.

15. Barlow DP, Stöger R, Herrman BG, Saito K, Scheifer N. The mouse IGF-II receptor is imprinted and closely related to the Tme locus. Nature $1991 ; 349: 84-7$.

16. Haig D, Graham C. Genomic imprinting and the strange case of the insuline-like growth factor II receptor. Cell 1991; 64 : 1045-6.

17. De Chiara TM, Robertson EJ, Efstratiadis A. A growth deficiency phenotype in hetcrozygous mice carrying an IGF-II gene disrupted by targeting. Cell 1991; 64: 849-59.

18. Lobel P, Fujimoto K, Ye RD, Griffiths $G$, Kornfeld S. Mutations in the cytoplasmic domain of the $275 \mathrm{kd}$ mannose 6-phosphate receptor differentially alter lysosomal cnzyme sorting and endocytosis. Cell $1989 ; 57: 787-96$.

19. Matzner U, von Figura K, Pohlman R Expression of the two mannose 6-phosphate receptors is spatially and temporally different during mousc embryogenesis. Development 1992 ; 114 : 965-72.

20. Von Figura K, Hasilik A. Lysosomal enzymes and their receptors. Ann Rev Biochem 1986 ; 55 : 167-93.

21. Duncan JR, Kornfeld S. Intracellular movement of the two mannose 6-phosphate receptors : return to the Golgi apparatus. $J$ Cell Biol $1988 ; 106$ : 617-28.

22. Goda Y, Pfeffer SR. Selective recycling of the mannose 6-phosphate/IGF II receptor to the trans-Golgi network in vitro. Cell 1988 ; $55: 309-20$.

23. Griffiths G, Simons K. The trans-Golgi network : sorting at the exit site of the Golgi
$\left(G_{i-2}\right)$ par l'intermédiaire d'une séquence de 14 acides aminés située dans son domaine cytoplasmique [14] (figure 5, p. 155).

Le gène du grand récepteur murin a été localisé sur le locus Tme ( $T$ associated mother effect) du chromosome 17, dont la délétion cause la mort de l'embryon au $15^{\mathrm{e}}$ jour de gestation [15]. Des études génétiques récentes ont montré que ce gène est soumis à un effet d'cmpreinte génomique maternelle, c'est-à-dire qu'il ne s'exprime qu'à partir de l'allèle d'origine maternelle. Curieusement, le gène de l'IGF-II est également soumis à une empreinte génomique mais n'est actif que s'il est hérité du patrimoine génétique paternel (pour revue, voir [13] et $m / s n^{\circ} 1$, vol. 8 , p. 65). Ce phénomène d'empreintes génomiques opposées pourrait reflèter le rôle du grand récepteur dans la dégradation de l'IGF-II. Il établirait ainsi un équilibre entre la demande paternelle d'embryons forts, capables d'assurer la descendance (production d'IGF-II) et la demande de la mère privilégiant le nombre d'cmbryons et de portées (expression du grand récepteur) (pour revue, voir [16] et $\mathrm{m} / \mathrm{s} n^{\circ} 3$, vol. 7, p. 292). Il est cependant surprenant que la délćtion du locus Tme soit létale, contrairement à l'inactivation du gène IGF-II [17]. Il est donc possible que ce récepteur ait d'autres fonctions importantes non caractérisées ou que d'autres gènes du locus Tme soient essentiels.

\section{Fonctions des récepteurs M6P dans le transport des ligands}

La raison pour laquelle les cellules possèdent deux récepteurs $\mathrm{M} 6 \mathrm{P}$ est mal comprise. Sont-ils redondants afin de garantir un bon fonctionnement des lysosomes ou assurent-ils des fonctions différentes dans le transport des hydrolases?

Le grand récepteur joue assurément un rôle majeur dans la rétention intracellulaire des hydrolases. Des lignées cellulaires établies (en général des macrophages), dépourvues de ce récepteur mais exprimant le petit, sécrètent $\simeq 70 \%$ de leurs hydrolases nouvellement synthétisées. L'expression du grand récepteur M6P dans ces cellules corrige cette hypersécrétion [18].

La fonction du petit récepteur est beaucoup moins claire car des mutants naturels dépourvus de ce récepteur n'ont pu être caractérisés. Cependant, des expériences de surexpression dans différents systèmes cellulaires suggèrent qu'il est à la fois impliqué dans la rétention intracellulaire des hydrolases et dans leur sécrétion (pour revue, voir $[8,13]$ ). Cette dernière possibilité est attrayante puisque la sécrétion d'hydrolases est souvent évoquée dans le remodelage des tissus embryonnaires et adultes ou dans la métastase de cellules cancéreuses. Il est aussi intéressant de noter que les récepteurs présentent des profils d'expression spatio-temporels très différents au cours de l'embryogenèse [19]. Nous avons récemment obtenu des souris transgéniques dont le gène codant pour ce récepteur a été inactivé par recombinaison homologue (Ludwig et al., non publié). L'obtention d'animaux viables exclut la participation du petit récepteur du M6P à l'embryogenèse (sécrétion d'hydrolases) ct devrait permettre d'établir sans ambiguïté la fonction de cette protéine.

Le grand récepteur présent à la surface cellulaire participe à l'endocytose de l'IGF-II, des hydrolases et, éventuellement, à celle d'autres ligands phosphorylés tels que le TGF $\beta$ et la proliférine (pour revue, voir $[8,13]$ ). Bien que présent et internalisé par endocytose, le petit récepteur n'est pas fonctionnel dans les conditions trouvées à la surface cellulaire.

\section{Transit intracellulaire des récepteurs M6P}

Différents modèles de transport des enzymes lysosomiales ont été proposés [20]. Cependant, les travaux les plus récents font apparaître que la grande majorité du transit est intraccllulaire et que les récepteurs déversent leurs ligands dans des compartiments prélysosomiaux (pour revue, voir [5, 8]) (figure 4).

Il est maintenant admis que le tri des enzymes lysosomiales s'effectue dans le réseau trans-golgien. Les oligosaccharides des hydrolases sont parfois sialylés, traduisant leur passage dans 
ce compartiment où la sialyltransférase est localisée (figure 1). De plus, les récepteurs présents à la surface cellulaire retournent à ce compartiment plus fréquemment $(\mathrm{t} 1 / 2 \simeq$ 2-3 heures) qu'aux citernes précoces de l'appareil de Golgi [21, 22]. Cette étape de tri nécessite la formation de vésicules mantelées par la clathrine qui bourgeonnent du réseau transgolgien [23].

Les hydrolases sont ensuite dirigées vers le système endocytique. Ce der- nier est composée : (1) d'endosomes précoces, organisés en réseau dynamique et spécialisés dans le recyclage des récepteurs de surface; (2) d'endosomes tardifs où sont localisés 80-90\% des grands récepteurs du M6P [24] (pour revue, voir [25, 26]). Cette localisation a souvent été interprétée comme traduisant un transport direct entre le Golgi et les endosomes tardifs. Néanmoins, nos résultats récents montrent que les endosomes précoces constituent le premier point de convergence entre les voies de transport exocytique et endocytique ([27], Méresse et Hoflack, non publié). Les endosomes tardifs représenteraient un compartiment où les récepteurs seraient stationnés avant d'être recyclés vers le réseau transgolgien. Une fois dissociées de leurs récepteurs, les hydrolases sont dirigées et stockées dans les lysosomes. L'endocytose des récepteurs présents sur la membrane plasmique requiert aussi la formation de vésicules man-

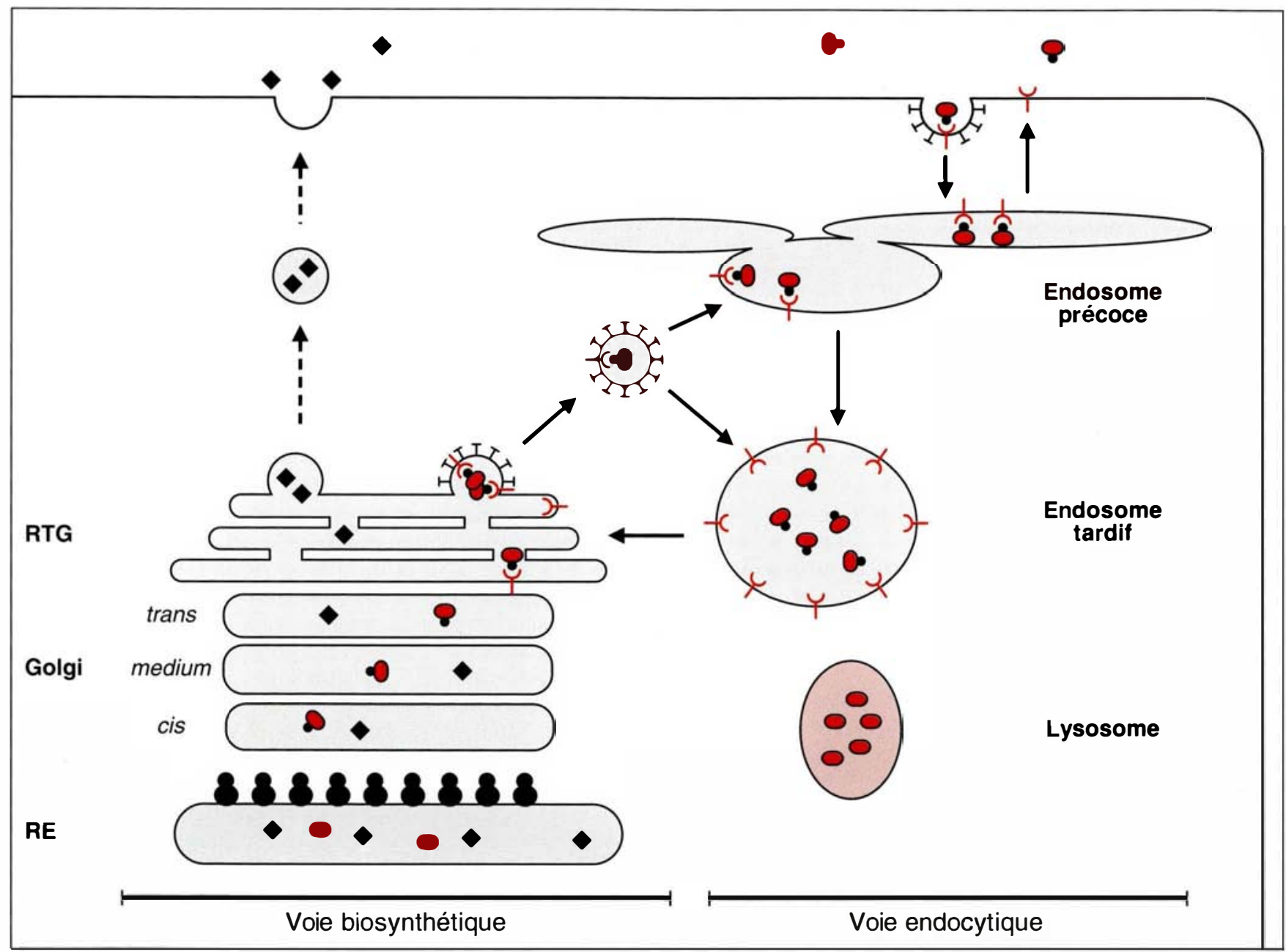

Figure 4. Modèle de transit intracellulaire des récepteurs du M6P. Les enzymes lysosomiales (O) et les protéines de sécrétion $(0)$ sont synthétisées dans le réticulum endoplasmique puis transportées vers l'appareil de Golgi où les hydrolases acides acquièrent le signal mannose 6-phosphate (O). Celui-ci permet la fixation des hydrolases aux deux récepteurs du mannose 6-phosphate Y dans le réseau trans-golgien (RTG). Les autres protéines suivent la voie de sécrétion. Les complexes enzyme/récepteur du M6P (T) sont empaquetés dans des vésicules mantelées par la clathrine qui bourgeonnent du réseau trans-golgien (RTG). Ces véhicules fusionnent ensuite avec les endosomes. Le pH bas des endosomes tardifs favorise la dissociation des complexes. Les hydrolases sont dirigées et stockées dans les lysosomes ou elles sont déphosphorylées. Les récepteurs retournent vers le réseau trans-golgien pour accomplir de nouveaux cycles de transport. Les récepteurs sont aussi présents sur la membrane plasmique où ils sont internalisés par des vésicules mantelées par la clathrine et dirigés vers les endosomes précoces. Ils sont ensuite recyclés soit vers la membrane plasmique soit vers le réseau trans-golgien. Les flèches pleines représentent le trajet des récepteurs et la flèche en pointillés la voie de sécrétion. 


\section{RÉFÉRENCES}

24. Griffiths G, Hoflack B, Simons K, Mellman I, Kornfeld S. The mannose 6 -phosphate receptor and the biogenesis of lysosomes. Cell 1988 ; 52 : 329-41.

25. Griffiths G, Grucnberg JE. The arguments for pre-existing early and late cndosomes. Trends Cell Biol 1991; 1: 5-9.

26. Murphy RF. Maturation models for endosomes and lysosome biogenesis. Trends Cell Biol 1991; 1 : 77-82.

27. Ludwig 'T, Griffiths G, Hoflack B. Dis tribution of newly synthesized lysosomal enzymes in the endocytic pathway of NRK cclls. J Cell Biol 1991; 115 : 1561-72.

28. Vaux D. The structure of an endocytosis signal. Trends Cell Biol 1992 : 2 : 189-92.

29. Jadot M, Canfied WM, Gregory W, Kornfeld S. Characterization of the signal for rapid internalization of the bovine mannose 6-phosphate/insuline-like growth factorII receptor. J Biol Chem 1992 ; 267 : 11069-77.

30. Johnson KF, Chan W, Kornfeld S Cation-dependent mannose 6-phosphate reccptor contains two internalization signals in its cytoplasmic domain. Proc Natl Acad Sci USA $1990 ; 87$ : 10010-4

31. Collawn JF, Kuhn LA, Sue Liu LF Tainer JA, Trowbridge IS. Transplanted LDI, and mannose 6-phosphatc receptor internalization signal promote high-cfficiency endocytosis of the transferrin receptor $E M B O$ J $1991 ; 10$ : 3247-53.

32. Mćresse $\mathrm{S}$, Ludwig $\mathrm{T}$, Frank $\mathrm{R}$, Hoflack B. Phosphorylation of the cytoplasmic domain of the bovine cationindependent mannose 6-phosphate receptor. $J$ Biol Chem 1990 ; 265 : 18833-42.

33. Pearse BMF, Robinson MS. Clathrin, adaptors and sorting. Ann Rev Cell Biol $1990 ; 6: 151-71$.

34. Beltzer JP, Spiess MS. In vitro binding of the asialoglycoprotein receptor to $\beta$ adaptin of the plasma membrane. EMBO J $1991 ; 12: 3735-42$.

35. Klionsky DJ, Herman PK, Emr SD. The fungal vacuole : composition, function and biogenesis. Microbiol Rev 1990; 54 : 266-92.

36. Mćresse S, Hoflack B. Phosphorylation of the cation-independant mannose 6-phosphatc receptor is closely associated with its exit from the trans-Golgi network. J Cell Biol 1993; 120 (sous presse). telées par la clathrine. Ces récepteurs de surface sont en équilibre rapide avec les récepteurs intracellulaires. Il n'existe donc qu'une population fonctionnelle de récepteurs [21]. Enfin, contrairement à d'autres classes de récepteurs dont l'endocytose est déclenchée par la liaison du ligand, les récepteurs du mannose 6-phosphate sont recyclés de façon constitutive entre les différents compartiments qu'ils visitent [21].

\section{Les mécanismes moléculaires du tri des récepteurs du M6P}

Des mécanismes de transport vésiculaire assurent le départ (et le retour) des récepteurs du réseau trans-golgien, de la membrane plasmique et des endosomes. Les étapes fondamentales de ces mécanismes sont : (1) le tri et la concentration des récepteurs dans une vésicule de transport; (2) la reconnaissance et la fusion de cette dernière avec la membrane cible.

Un développement récent de la biologie cellulaire a été la découverte des signaux de tri des réccptcurs (pour revue, voir [5, 8, 28]). L'expression de mutants du grand récepteur a permis de montrer qu'une séquence de quatre acides aminés de son domaine cytoplasmique $\left(\mathrm{Y}^{26} \mathrm{SKV}^{29}\right)$, contenant une tyrosine critique, est nécessaire à son endocytose [18, 29] (figure 5). Cette séquence peut être déplacée à l'intérieur du domaine cytoplasmique ou substituée par les motifs d'endocytose d'autres récepteurs (par exemple, ceux des LDL ou de la transferrine). Le petit réccpteur contient, quant à lui, deux signaux d'endocytose (Phe ${ }^{13}$ et $\mathrm{Phe}^{18}$, et $\mathrm{Y}^{45} \mathrm{RGV}^{48}$ ) [30]. Des prédictions de structures (figure 6 ) puis des études de RMN ont montré que ces motifs d'endocytose adoptent tous une conformation de boucle serrée (tight turn) ([31], pour revue, voir [28]). Cette conformation est en fait plus importante que la nature des acides aminés qui la compose puisque les substitutions conservatives restent fonctionnelles.

Les signaux permettant la sortie sélective des récepteurs du réseau trans-golgien et des endosomes sont moins bien définis. Lobel et al. [18] ont cependant montré que la partie C-terminale du grand récepteur est nécessaire à la rétention intracellulaire des hydrolases. Celle-ci est en fait la résultante de tris successifs dans le Golgi et les endosomes. Cette région du domaine cytoplasmique contient deux séquences d'acides aminés extrêmement conservées (figure 5). Nous avons montré que deux sérines à l'intérieur de ces séquences (résidus 85 et 156) sont phosphorylées [32] lors du transport du récepteur entre le Golgi et les endosomes, puis rapidement déphosphorylées dans les endosomes [36]. S'il est encore difficile d'évaluer le rôle de ces phosphorylations, ce résultat suggère que ces sites sont impliqués dans le transit intracellulaire du grand récepteur. L'autre récepteur contient également un site de phosphorylation très similaire dans sa partie C-terminale. La mutagenèse dirigée de ce site indique qu'il détermine la redistribution de ce récepteur entre les structures endocytiques précoces et tardives, et suggère qu'il est impliqué dans la retention intracellulaire des hydrolases (Mauxion et Hoflack, non publié).

Des protéines cytosoliques reconnaissent ces signaux et favorisent la concentration des récepteurs dans des vésicules de transport. Les adaptateurs (protéines d'assemblage) semblent jouer ce rôle (pour revue, voir [33]). La sous-unité $100 \mathrm{kDa}$ de ces complexes de quatre protéines (110, $100, \approx 50$ et $\approx 20 \mathrm{kDa}$ ) favorise aussi la polymérisation de la clathrine sur le réseau trans-golgien ou sur la membrane plasmique, facilitant ainsi le bourgeonnement de vésicules. Des études in vitro ont montré que les adaptateurs HA-II, spécifiques des vésicules mantelées d'endocytose, se fixent sur le motif d'endocytose de nombreux recepteurs, y compris celui du grand récepteur du mannose 6-phosphate. En revanche, les adaptateurs HA-I, spécifiques des vésicules issues du Golgi, interagiraient avec un autre motif présent dans le domaine cytoplasmique du grand récepteur du mannose 6-phosphate (pour revue, voir [33]). Le rôle des différentes sous-unités de ces complexes reste difficile à évaluer. La sous-unité de $100 \mathrm{kDa}$ de HA-II pourrait lier les récepteurs [34], la sous-unité de $50 \mathrm{kDa}$ de $\mathrm{HA}$-I serait la kinase modifiant le domaine cytoplasmique du grand récepteur du mannose 6-phosphate [32]. Cepen- 


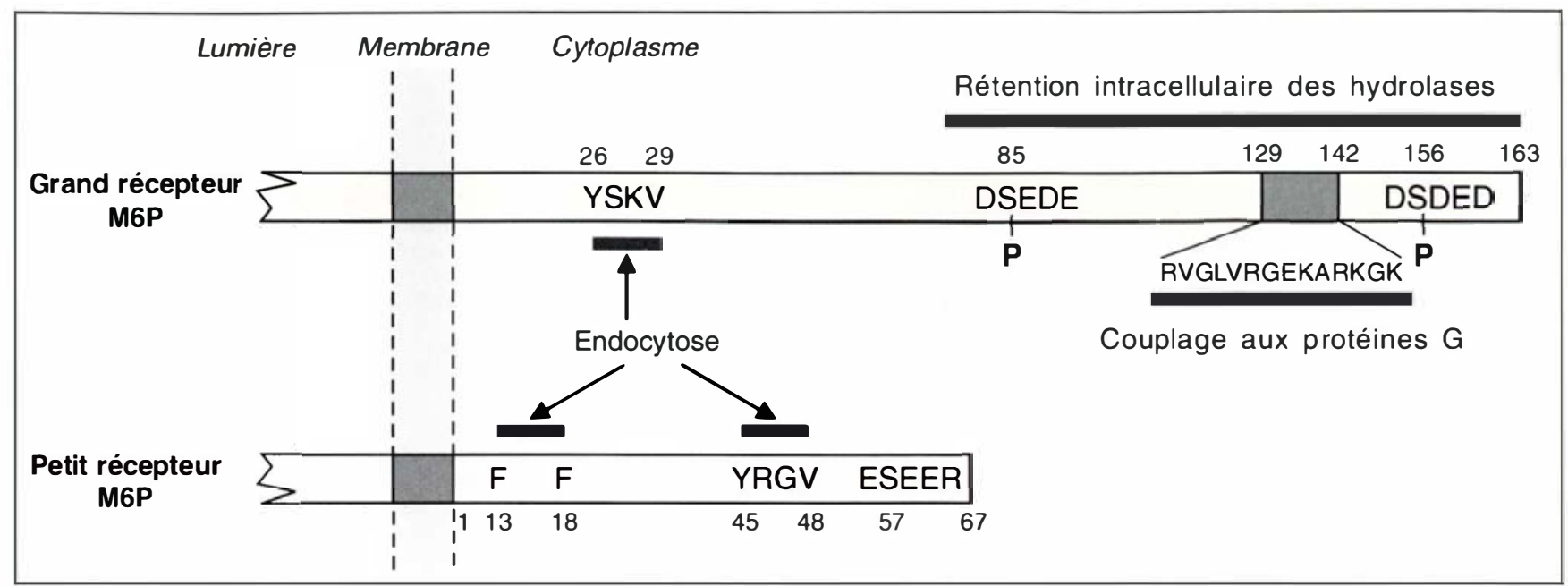

Figure 5. Les domaines cytoplasmiques des récepteurs du M6P contiennent de multiples signaux. La séquence $Y^{26}{ }^{2} K V^{29}$ du domaine cytoplasmique du grand récepteur du M6P constitue le motif d'endocytose. La moitié Cterminale du domaine cytoplasmique est nécessaire au transport des hydrolases vers les lysosomes. Cette moitié renferme deux domaines conservés contenant des sites de phosphorylation pour les caséine kinases de type II. Ces sites sont phosphorylés sur les sérines (résidus 85 et 156) lors du transport du récepteur entre le Golgi et les endosomes. Cette partie du domaine cytoplasmique contient également une séquence de 14 résidus (129 à 142) qui présente des analogies avec le mastoparan, un peptide activateur des protéines $G$ trimériques. Le domaine cytoplasmique du petit récepteur contient deux motifs d'endocytose (résidus de phénylalanine 13 et 18 et la séquence Y45RGV ${ }^{48}$ ) ainsi qu'un site de phosphorylation potentiel (S57).

dant, d'autres études sont nécessaires pour confirmer l'importance des adaptateurs dans le tri des récepteurs.

\section{Conclusion et perspectives}

Des progrès considérables ont été accomplis au cours de ces dernières années dans la compréhension des mécanismes moléculaires impliqués dans le transport des hydrolases vers les lysosomes. Sans nul doutc, ceuxci devraient contribuer à l'amélioration des thérapies des maladies lysosomiales [2]. Il est très probable que le clonage de l'ADNc et du gène de la phosphotransférase permettra non sculement d'établir les bases génétiques des mucolipidoses II et III, mais aussi de mieux comprendre les mécanismes fondamentaux des interactions entre cette enzyme clé et ses différents substrats. Enfin, la détermination de la structure tridimensionnelle de plusieurs enzymes lysosomiales devrait également contribuer à mieux comprendre comment cette classe de protéines est spécifiquement reconnue par cette enzyme.

$m / s n^{\circ} 2$ vol. 9, février 93

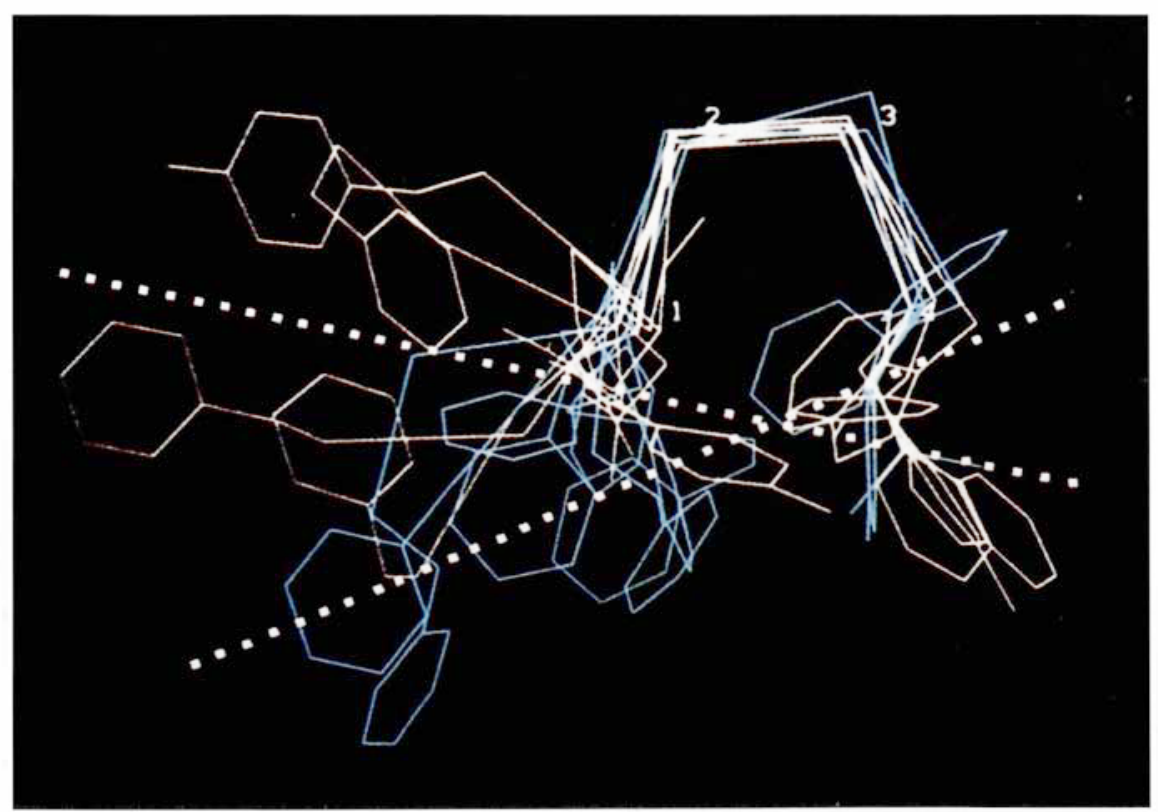

Figure 6. Conformation du motif d'endocytose. Les motifs d'endocytose du grand récepteur du M6P $\left(Y^{-2} X Y^{1} X K V^{4}\right)$ et du récepteur des $L D L$ $\left(F^{-2} X N^{1} P X Y^{4}\right)$ ont été comparés à des séquences analogues de protéines de structure connue. Les résidus 1 à 4 des conformères du récepteur des LDL (jaune) et du récepteur du M6P (bleu) forment une boucle serrée ( $\beta$ turn). Les chaînes latérales des résidus - 2, 1 et 4 du motif d'endocytose des deux récepteurs sont alignées (lignes pointillées. (D'après [31].) 
La présence de deux récepteurs du mannose 6-phosphate et la dualité de ligand du récepteur M6P/IGF-II restent à expliquer. L'étude du transport de ces molécules contribuera également à mieux comprendre leur fonction. Les mécanismes moléculaires mis en jeu, en particulier lors de l'endocytose, commencent à se dessiner. Les motifs et les machineries assurant les autres étapes de tri restent encore à définir. D'autres questions restent sans réponse : comment les adaptateurs du Golgi et de la membrane plasmique reconnaissent-ils leur membrane cible ? Comment ces interactions sont-elles réglées? Enfin, existe-il d'autres complexes protéiques, analogues aux adapteurs, impliqués dans le recyclage des récepteurs vers le Golgi et la membrane plasmique?

Nous ne saurions terminer cette revue sans mentionner l'existence probable d'autres systèmes de ciblage vers les lysosomes. C'est le cas, par exemple, de la phosphatase acide ou de la $\beta$-glucocérébrosidase dont le déficit caractérise la maladie de Gaucher. La phosphatase acide, synthétisée sous une forme transmembranairc et non phosphoryléc, est ciblée vers les compartiments endocytiques de manière analoguc aux récepteurs du mannose 6-phosphate. Elle est ensuite clivée et relachée sous forme soluble dans les lysosomes. Les hydrolases solubles pourraient également utiliser un mécanisme de ciblage indépendant du signal M6P [5]. Dans la mucolipidose II, où le signal M6P n'est pas synthétisé, certaines cellules (les hépatocytes, par exemple) possèdent des taux normaux de certaines hydrolases. Une explication séduisante de cc phénomène pourrait être trouvée chez la levure. Cct cucaryote inféricur ne synthétise pas le signal M6P, n'exprime pas les récepteurs du mannose 6-phosphate et cible ses hydrolases vers la vacuole (lc lysosome) par un mécanisme indépendant du mannose 6-phosphate. Chez la levure, le signal de tri est probablement contenu dans la partie protéique des enzymes vacuolaires [35]. Il est possible que ces mécanismes de transport aicnt été maintenus au cours de l'évolution et contribuent aussi à assurer lc bon fonctionnement des lysosomes

\section{Summary}

Molecular basis of transport to lysosomes

In high eucaryotes, the mannose 6-phosphate receptors (MPRs) are essential components that allow proper targeting of lysosomal enzymes to lysosomes. Two mannose 6-phosphate receptors have been characterized. The first has a dual function. It binds the phosphorylated oligosaccharides of the lysosomal enzymes and the insulin-like growth factor II, a non-glycosylated polypeptide. The second only binds lysosomal enzymes. It is still unclear why mammalian cells need two different MPRs that apparently show similar targeting functions. The MPRs are found in scveral intracellular compartments. They form however one functional pool of receptors. They recycle between the transGolgi network and the endocytic compartments to divert the newly synthesized lysosomal enzymes from the secretory pathway. They also recycle between the plasma membrane and the endosomes for internalization of extracellular ligands. At steady state, the MPRs are mostly localized to endosomes where ligands dissociate before being transported to the lysosomes. These steps of transport, some of which involving the formation of clathrin-coated vesicles require signals contained in their cytoplasmic domains. These latter interact with cytosolic components which allow the segregation and clustering of the MPRs into transport vesicles.

S. Méresse a bénéficié d'une allocation de recherche de la CEE (Science Plan, contrat $n^{\circ}$ 1000316).

\section{TIRÉS A PART}

B. Hoflack.
A l'occasion du Solon AUTONOMIC 93 et des manifestations du SETAA (Services et Techniques pour Aveugles ef Amblyopes), un séminaire sur le thème " Présentations non-visuelles dans la communication hommeordinateur - Accès aux représentations figuratives pour les personnes handicapées de la vue "I se tiendra à Paris (MRE) les 29 et 30 mars 1993 avec, entre autres, le soutien de I'INSERM.

Comité d'organisation : D. Burger (INSERM U.BB), J.-C. Spérandio (Université Paris VI), M. Goldberg (INSERM U.BB), J.-L. Joulia (SETAA-ANPEA, Association Nationale des Parents d'Enfants Aveugles), Y. Burnod (INSERM, CNRS URA 1199)

Comité scientifique : $S$. Aymé (INSERM S.C.11), Ph. Barker (G.B.), D. Béroule (CNRS), Ch. Fluhr (CEA), Y. Hatwell (Université Grenoble II), A. Rousseau (CNRS), U. Strempel (Allemagne).

L'objectif de ce séminaire, organisé dans un esprit pluridisciplinaire, est de faire un tour d'horizon complet des recherches en cours sur les interfaces non-visuelles. L'accent sera mis sur : - Les représentations figuratives dans la conduite des activités intellectuelles; $\bullet$ La substitution d'un système de présentation à un autre ; - Les techniques de présentation multimodales ; $\bullet$ Les architectures logicielles permettant le développement d'interfaces multimodales:- De la standardisation des objets et des méthodes non-visuelles; $\bullet$ L'apport de l'intelligence artificielle dans les systèmes $d$ 'interfaces.

Ces journées sont destinées aux "développeurs ", aux chercheurs et à tous les professionnels concernés par l'amélioration des systèmes $d^{\prime}$ interface et la plus grande accessibilité des produits informatiques aux personnes handicapées, en particulier.

\section{Programme}

- Images et cognition (M. Denis, LIMSI-CNRS F.) : - Images et représentations mentales chez l'aveugle (Y. Hatwell, Université Grenoble, II) ; - Communication pictographique (P. Barker GB) :- Images tactiles et audio-tactiles (R. Hinton, GB) ; - Apprendre avec des objets auditifs et tactiles (D. Burger, INSERM et C. Mazurier, CNEFEI) : - Interactions homme-machine multimodales (D. Béroule et J.-C. Martin, CNRSLIMSI ; - Manipulation directe des données (A. Robby-Brami, INSERM); - Analyse des scènes et descriptions linguistiques (Y. Burnod, INSERM); - Représentation des textes (C. Fluhr, INSTN-CEA) ; • Accès amélioré aux quotidiens, livres et dictionnaires électroniques (A. Schwindling et U. Strempel, Allemagne); - Représentations mathématiques : figures, formules et courbes (A. Edwards, GB) ; - Accès aux interfaces en modes graphiques (J. Berliss, USA) ; - Objets, méthodes et standards d'interaction non-visuels (J.-C. Spérandio, Université Paris V).

Les actes paraîtront pour le séminaire, sous forme d'une co-édition INSERM/John Libbey Eurotext dans la collection des Colloques de I'INSERM. Pour renseignement complémentaire et inscription, s'adresser de toute urgence a : ANPEASETAA, 12 bis, rue de Picpus, 75012 Paris Tél. : 43.42.40.40 - Télécopie : 43.42.40.66. 